\title{
Search for Ultra-low Mass Dark Matter
}

\section{L.Chevalier*.}

IRFU, CEA, University Paris-Saclay, F-91191 Gif-sur-Yvette, France

E-mail: laurent.chevalier@cea.fr

\begin{abstract}
The absence of observation of dark matter candidates above the GeV scale, at the LHC and in dedicated WIMP search experiments, led physicists to explore other mass domains, in particular below the electron-volt. Beside, the so-called strong CP problem is still an issue in the standard model. It can be solved by adding a new scalar field called axion. This scalar field could be a dark matter candidate. Depending of its coupling and in the range of mass $\approx\left[10^{-22}, 1\right] \mathrm{eV}$, it may solve the two issues described above at once. After the description of the strong $\mathrm{CP}$ problem and its simplest solution, I briefly present some mass limits for axions and similar fields (Axion Like Particle, Hidden Photon) and some of the experiments from which these limits have been extracted. At the end of this document, new limits on Hidden Photon are presented with a new experiment: Shuket.
\end{abstract}

2nd World Summit: Exploring the Dark Side of the Universe

25-29 June, 2018 - EDSU2018

University of Antilles, Pointe-à-Pitre, Guadeloupe, France

${ }^{*}$ Speaker. 


\section{Introduction}

One of the main problem in fundamental physics is the nature of the dark matter which would constitute $85 \%$ of the matter of the universe. Many different solutions, beyond the standard model, have been proposed to solve this problem. New particles have been hypothesized like Lightest Supersymmetric Particle or Weakly Interacting Massive Particles. But despite intensive searches, at the LHC [1, 2] ${ }^{1}$ and in dedicated experiments for WIMPs [3], no experimental clue has been found. Among all these hypotheses, for what dark matter could be, a new scalar field, the axion, has been hypothesized in the late seventies and extensively searched for ever since. The best lower mass limit is given by Planck results [4].

$$
\frac{\lambda_{\mathrm{dB}}}{2 \mathrm{kpc}} \sim\left(\frac{10^{-22} \mathrm{eV}}{\mathrm{m}_{\mathrm{a}}}\right)\left(\frac{10 \mathrm{~km} / \mathrm{s}}{\mathrm{v}}\right)
$$

Likewise, studies of the galaxy structure formation provide a lower bound constraint, a simple estimation of the minimal wavelength of axion as a dark matter candidate led to a mass limits about $10^{-22} \mathrm{eV}$, see equation 1.1. $\lambda_{d B}$ is the de DeBroglie wavelength corresponding to a typical dwarf galaxy diameter and typical velocity. An upper bounds on the mass of the axion $\mathrm{QCD}^{2}$ has been set around one electron-Volt from cosmological considerations and observations of the sun, see for instance [5].

The first consequence with this mass range is the character of the axion field. The equation 1.2, with $N_{\text {particles }}$ is an estimation of the number of particles of dark matter in a standard galaxy and $N_{\text {cells }}$ is an estimation of the numbers of cells in phase space. The ratio of these two numbers shows that a dark matter candidate with a mass below $100 \mathrm{eV}$ and $a$ fortiori for $1 \mathrm{eV}$, must be a boson. At last, three types of potential dark matter candidates are presented, depending on the hypotheses on their coupling. The axion $\mathrm{QCD}$ has a coupling constant proportional to its mass and can be observed through the Primakoff process. The Axion Like Particle or ALP, a scalar field dark matter candidate but not strong CP problem solution, has a coupling constant to photon not proportional to its mass but as the axion, can be signed in presence of a magnetic field. Finally, Hidden Photon or HP is a field with a coupling constant to photon which is not proportional to its mass and can be observed without magnetic field.

$$
\left.\begin{array}{l}
N_{\text {particles }} \sim \frac{10^{12} M_{\odot}}{m} \\
N_{\text {cells }} \sim \frac{\frac{4 \pi}{3} p_{\max \frac{3 \pi}{3} R^{3}}^{(2 \pi)^{3}}}{{ }^{3}}
\end{array}\right\} \Rightarrow \frac{N_{\text {particles }}}{N_{\text {cells }}} \sim\left(\frac{100 \frac{\mathrm{eV}}{\mathrm{c}^{2}}}{m}\right)^{4}
$$

\section{The strong-CP problem}

The so-called "strong CP problem" emerged in the middle of the seventies with S.Weinberg observation; there is no $\mathrm{U}(1)_{\text {axial }}$ symmetry in the strong interaction while the chiral symmetry ${ }^{3}$ is almost preserved. One can summarize the main four stages $[6,8,9,10]$ which led to the simplest solution:

\footnotetext{
1 for instance on supersymmetry and its natural dark matter candidate the LSP and more generally most of direct searches with missing energy in the final state.

2 axion solution of the strong-CP problem

$3 \mathrm{~m}_{\text {quark } \rightarrow 0} \Rightarrow$ strong interraction invariant by $\mathrm{U}(2)_{\text {vector }} \mathbf{x} \mathrm{U}(2)_{\text {axial }}$

$\mathrm{U}(2)_{\text {vector }}=\mathrm{SU}(2)_{\text {vector }} \mathbf{x} \mathrm{U}(1)_{\text {vector }} \Rightarrow$ isospin $\mathbf{x}$ baryon is almost symmetry of nature

$\mathrm{U}(2)_{\text {axial }}$ spontaneously broken symmetry but no sign of another light state in the hadronic spectrum...
} 
1. S.Weinberg: defined the $\mathrm{U}(1)_{\text {axial }}$ problem

$\rightarrow$ in massless quarks approximation, chiral symmetry is respected by the Lagrangian

$\rightarrow$ baryonic number conservation $\Rightarrow \mathrm{U}(1)_{\text {vector }}$ good approximate symmetry of nature

$\rightarrow$ no associated pseudo-Goldstone boson observed around the pion mass $\Rightarrow \mathrm{U}(1)_{\text {axial }}$ is not a symmetry of the strong interaction

2. G. 't Hooft: provided a explanation of this problem directly linked to anomalies

$\rightarrow$ add a full derivative term to the Lagrangian it affects neither the equations of motion, nor the perturbative aspects of the theory

$\rightarrow$ this term break explicitly $\mathrm{U}(1)_{\text {axial }}$ $\Rightarrow \mathrm{QCD}$ vacua are degenerate

$\Rightarrow$ term violates $\mathrm{P}$ and $\mathrm{T}$ while $\mathrm{CPT}$ is conserved $\Rightarrow$ strong CP-violation

3. R.Pecci-H.Quinn: no experimental indication of strong CP-violation, propose a solution

$\rightarrow$ it exists a new global symmetry $\mathrm{U}(1)_{\mathrm{PQ}}$, spontaneously broken

4. S.Weinberg and F.Wilczek:

$\rightarrow$ the new global symmetry leads to a new light scalar field called axion

On the one side, the QCD vacua structure impose a term which violates the strong CP symmetry. This added and mandatory term to the standard model Lagrangian is given by the equation 2.1. This term is normalized by a new free parameter, called $\theta . \alpha_{s}$ is the dimensionless strong structure constant and the "a" indices are running on the color charges. On the other side the Electric Dipole Moment of the neutron is measured to be under $2.910^{-26} \mathrm{e} . \mathrm{cm}$ [11]. This measurement implies an upper bound on $\theta$ of $10^{-10}$. Such a so tiny value for the $\theta$ parameter is always possible but it seems also reasonable to assume that is a consequence of an unknown global symmetry spontaneously broken, here $\mathrm{U}(1)_{\mathrm{PQ}}$ and its associated scalar field. This new scalar field solve the strong $\mathrm{CP}$ problem by forcing $\theta$ value to dynamically tend to zero. Beside this description of the pure QCD effect, $\theta$ can be interpreted as a fine tuning in the standard model. It can be reinterpreted as a sum of two CP-violation effects. One coming from the QCD vacua previously described and second coming from the well known weak CP-violation through an additional phase in the CKM matrix in the standard model. The neutron EDM is sensitive to the sum of these two effects, so why $\theta$, coming from two completely different mechanisms is so close to zero?

$$
\mathscr{L}_{\theta}=\theta \frac{\alpha_{s}}{8 \pi} G_{\mu \nu}^{(a)} \tilde{G}^{\mu v(a)} \rightarrow \mathscr{L}_{a}=\frac{a(x)}{f_{a}} \frac{\alpha_{s}}{8 \pi} G_{\mu \nu}^{(a)} \tilde{G}^{\alpha \beta(a)}
$$

The equation 2.1 shows the replacement of the $\theta$ parameter by a new term, a(x) which dynamically relax to zero and consequently induce no more CP-violation. $f_{a}$ is the scale at which $\mathrm{U}(1)_{\mathrm{PQ}}$ symmetry is spontaneously broken. Some remarks; the non-pertubative QCD effect around a temperature close to $\lambda_{\mathrm{QCD}}$ led to an explicit symmetry breaking also called misalignment and consequently the axion pseudo-Goldstone boson becomes massive. For $f_{a}$ around $246 \mathrm{GeV}$, the axion mass should be around $100 \mathrm{keV}$. But the beam-dump experiment [12] has ruled out this solution. Nevertheless the U(1) $)_{\mathrm{PQ}}$ symmetry breaking could happen at higher temperature, $f_{a} \gg 246 \mathrm{GeV}$. This solution is now privileged and effective models ${ }^{4}[13,14]$ propose the axion ${ }_{\mathrm{QCD}}$ as a dark matter candidate over an ultra low mass range $\approx\left[10^{-22}, 1\right.$. $] \mathrm{eV}$.

\footnotetext{
4 DZFS and KSFZ
} 


\section{Present limits on axion mass}

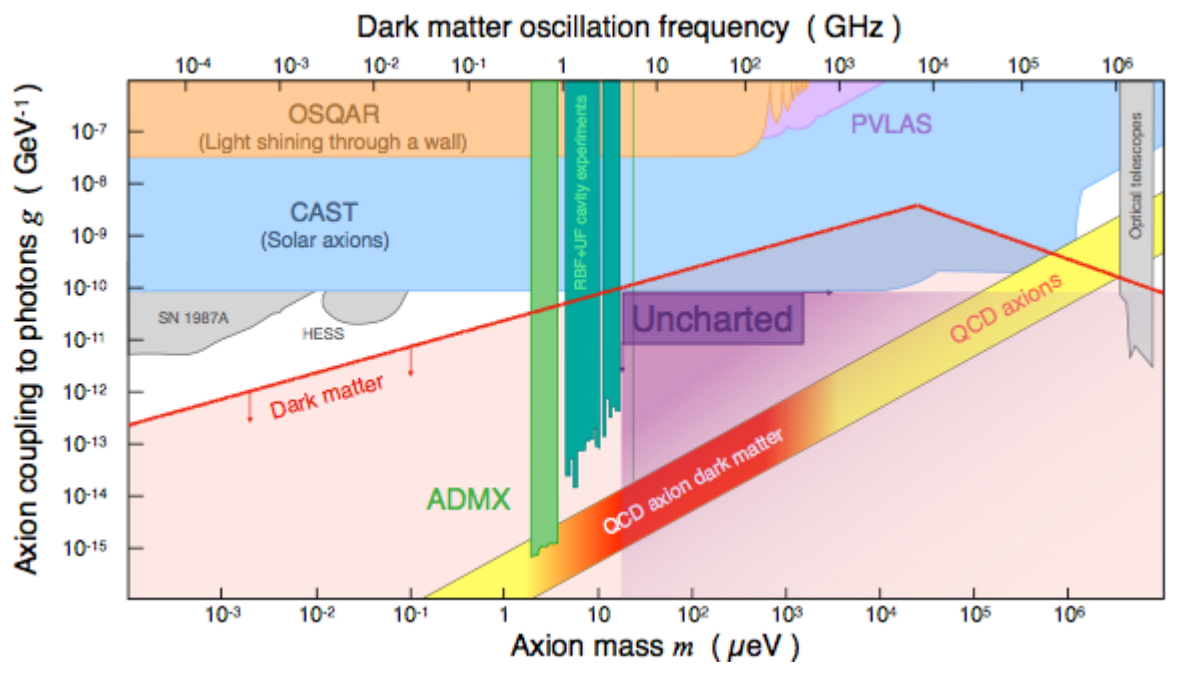

Figure 1: Parameter space for axion searches, coupling to photons as function of mass.

Figure 1 presents a review of current constraints on the parameter space for axion searches, coupling to photons as function of mass. The colored parts; gray, light blue, orange and green are already excluded by laboratory results, stellar physics, and cosmology-dependent arguments. The semi transparent purple light region below the red line corresponds to dark matter candidates which do not solve the strong CP problem. Finally the oblique yellow band is the hunted region of the parameter space where the solution to the strong-CP problem, axion ${ }_{\mathrm{QCD}}$ is a dark matter candidate. Two classes of models exist, in the pre-inflationary scenario, the PQ symmetry is broken before and during inflation and not restored afterwards. In this case, there is an upper bound on the mass of about $1000 \mu \mathrm{eV}$. In the post-inflationary scenario the mass range is more constrained and belong to the interval $\approx[50,500] \mu \mathrm{eV}$. Figure 1 corresponds to the post-inflationary scenario and inside the yellow band, a specific region has been colored in red, corresponding to this mass interval. The blank part, of this figure, is largely uncharted and correspond to the weaker constraints on ALPs and will be explored in the next years. It is a mandatory path to reach more theoretically well motivated region.

\section{Search strategies and future prospect}

In addition to many interpreted or direct limits, coming from astrophysical observations on stars evolution, galaxies, galaxy clusters or dwarf galaxy, there are in laboratory many different experiments which are now running and provide the best limits on the mass of the axion. All are using the Primakoff process. To detect photons, from interaction between the axion to magnetic field, many different physical mechanisms are used; antenna, gaseous detector, bolometer for instance. Due to the very low signal power, experiment called haloscope, use a cavity to reach a resonance and amplify the signal. For instance ADMX [17], see figure 1 and its present limits. AMDX is the only experiment sensitive to DFSZ model axion but over a narrow bandwidth. An other type 
of experiment called helioscope use a large volume with high magnetic field about $10 \mathrm{~T}$. The main helioscope is CAST [18], see also figure 1 for the current boundaries. Helioscopes are less sensitives in terms of coupling constant reachable but have a larger bandwidth and haloscope have a much better sensitivity on coupling constant but over a very small mass range. The future of these two types of experiment, IAXO [20] for CAST and ADMX-upgrade and Haystac [19] for ADMX, will improve by orders of magnitudes these limits. Many other new experiments are in preparation but the list is too long for this short note but can be found in the pdg [21]. Among all these new projects, MadMax is probably the most ambitious and a description can be found here [22]. Among this list, one can add a first dish experiment without magnetic field which is the first step to axion ${ }_{\mathrm{QCD}}$ detection and provide now first results on Hidden Photon: SHUKET (SearcH for U(1) darK matter with an Electromagnetic Telescope). This new experiment using a portion of a metallic sphere of $32 \mathrm{~m}$ diameter and a microwave detection setup. The results from a HP search campaign is presented in figure 2. The red lines show the first exclusion of HP around $3.10^{-5} \mathrm{eV}$. The next step is to reproduce the same measurement with a magnetic field to provide limits on axion.

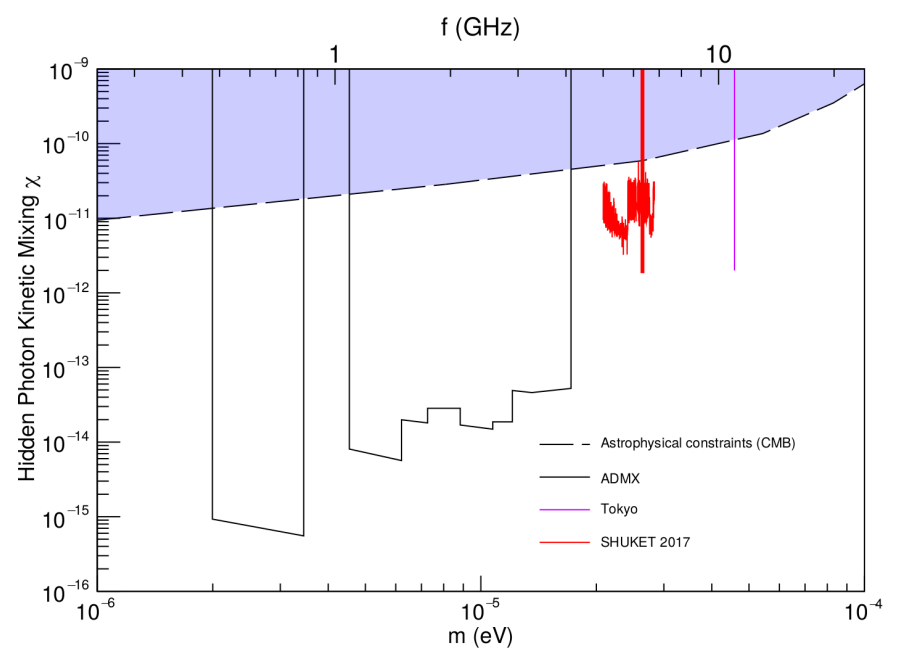

Figure 2: Shuket present results in a plane kinetic mixing versus mass. The blue part is already excluded region by cosmological constraints. Black lines are the ADMX and old measurements exclusions extrapolated from their present limits showed in figure 1. The red lines are the preliminary results from Shuket.

\section{References}

[1] ATLAS collab. JHEP1O(2015)134.

[2] ATL-PHYS-SLIDE-2018-728

[3] XENON collab. Phys. Rev. Lett. 119, 181301.

[4] R.Hlozek et al. arXiv:1708.05681

[5] S.Hannestad et al. JCAP 0404 (2004) 008.

[6] S.Weinberg, Phys. Rev. D11, 3583 (1975).

[7] G. 't Hooft, Phys. Rev. Lett. 37, 8 (1976); Phys. Rev. D14, 3432 (1976).

[8] D.Peccei and H.B.Quinn, Phys.Rev.Lett. 88,1440 (1977).

[9] S.Weinberg, Phys. Rev. Lett. 40,223 (1978).

[10] F.Wilczek, Phys. Rev. Lett. 40,279 (1978).

[11] C.A.Baker et al. Phys. Rev. Lett. 97, 131801 (2006).
[12] B. Dobrich, arXiv:1708.05776v1.

[13] KSFZ Phys.Rev.Lett.43,103(1979) and Nucl.Phys.B166,493(1980).

[14] DZFS Phys.Lett.B104,199(1981) and Sov. J.Nucl.Phys.31,260(1980).

[15] P.Arias et al., JCAP 1206 (2012) 013.

[16] D.Horns et al., JCAP 1304 (2013) 016.

[17] Stern, I. (2016). ADMX Status.

[18] CAST collab. Nature Phys. 13 (2017) 584-590.

[19] B.M.Brubaker et al., Phys. Rev. Lett. 118, 061302 (2017).

[20] IAXO, JINST9 (2014) T05002.

[21] PDG, Chin. Phys. C, 40, 100001 (2016).

[22] P. Brun et al., Madmax white paper 